\title{
Coherent radar reflections from an electron beam induced particle cascade
}

\author{
Steven Prohira* for the T-576 Collaboration ${ }^{\dagger}$ \\ Center for Cosmology and AstroParticle Physics, The Ohio State University, Columbus, $\mathrm{OH}$ \\ 43210 \\ E-mail: prohira.1eosu.edu
}

\begin{abstract}
Experiment T-576 ran at SLAC in 2018, in development of a new radar-based detection scheme for ultra-high energy neutrinos. In this experiment, the electron beam $\left(\mathrm{N} \sim 10^{9} e^{-}\right.$at $\left.\sim 10 \mathrm{GeV}\right)$ was directed into a plastic target to simulate a $10^{19} \mathrm{eV}$ neutrino-induced shower in ice. This shower was interrogated with radio frequency $(\mathrm{RF})$ radiation, in an attempt to measure a radar-like reflection from the ionization produced in the target during the particle shower. This technique could be employed to detect the rare interactions of ultra-high-energy neutrinos in dense material, such as polar ice sheets, extending the extant energy range of detected neutrinos up to $\mathrm{EeV}$ and beyond. In this proceeding, we detail the experiment and present results from the analysis and the observation of a signal consistent with a radar signal.
\end{abstract}

36th International Cosmic Ray Conference -ICRC2019-

July 24th - August 1st, 2019

Madison, WI, U.S.A.

\footnotetext{
* Speaker.

${ }^{\dagger}$ for collaboration list see PoS(ICRC2019)1177
} 


\section{Introduction}

Radio techniques are likely the key to detecting ultra-high energy (UHE) neutrinos $\left(\gtrsim 10^{16} \mathrm{eV}\right.$ ) due to inaccessibly low fluxes for existing, proven detector technologies such as IceCube [1]. This is because radio detectors can instrument large volumes with minimal apparatus, therefore making it feasible to build a detector with the tens to hundreds of cubic kilometers of requisite target volume to detect particles that may interact with the earth on the order of once per square kilometer per century.

When an UHE neutrino interacts in a target volume, which for modern detectors is generally ice or water, it produces a cascade of charged and neutral particles. Some radio detectors $[2,3,4,5]$ seek to detect the RF produced by these cascades through the Askaryan effect [6], which is a coherent emission of radio along the usual Cherenkov angle, from a negative charge excess that builds up within the cascade during progression. This emission scales with the square of the number of particles in the cascade, and so is an attractive option for very high energy cascades, even though it has a limited geometric acceptance due to the emission being limited to an $\mathscr{O}(1 \mathrm{deg})$ window around the Cherenkov angle.

An alternative approach is found by considering the ionization left behind as the high-energy cascade traverses the medium [7, 8, 9]. For UHE neutrinos that are observed in dense media, the density of this ionization trail will be high enough to reflect incident radio waves. Therefore, if a volume is instrumented with a transmitter to illuminate the volume and receivers to monitor it, an UHE neutrino cascade occurring within this volume will allow radio waves from the transmitter to be reflected to the receivers. These reflected waves are detectable at a much wider range of angles than Askaryan radiation, largely increasing the aperture for the detection method. Furthermore, by providing enough transmitter power, the method may allow for the detection of UHE neutrinos of lower energies just beyond the IceCube reach

The power of a returned radar signal from a neutrino induced cascade should scale linearly with the transmitter output power, therefore allowing for the effective lowering of the energy threshold through increasing transmitter power. This effective increase in the signal-to-noise ratio (SNR) through increased transmitter output power is not available to other detection schemes.

In this proceeding, we describe a recent test-beam experiment, T-576 at the SLAC National Accelerator Facility, which shows the first evidence for what may be a radar signal from a highenergy particle cascade. T-576 took 2 runs, in May and October of 2018, and this proceeding concerns the latter run. Details of the first run can be found in Ref. [10], and a more detailed analysis will follow in a forthcoming publication.

\section{Experimental setup}

The End Station A facility at SLAC has a storied history in particle physics [11] and radio astroparticle physics [12, 13, 14]. For experiment T-576, a 4 meter long target of high-density polyethylene (HDPE) was installed at the end of the beamline. Surrounding this target were 15 receiving antennas and a transmitting antenna, which was broadcasting a continuous-wave $(\mathrm{CW})$ radio signal at the target, aimed at the point of maximum energy deposition of the shower. The full $10 \mathrm{GeV} / \mathrm{e}$ beam was directed into this target at a rate of $1 \mathrm{~Hz}$. This beam contained roughly 


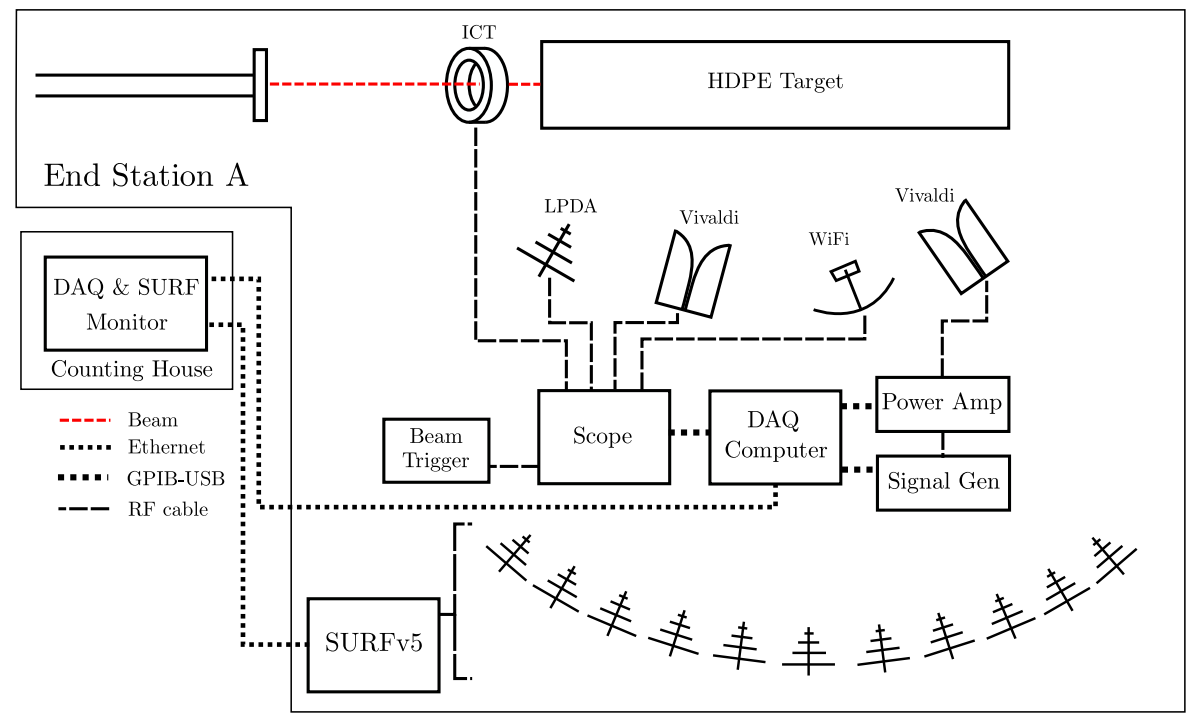

Figure 1: Experimental schematic for T-576.

$10^{9}$ electrons per bunch, making a single bunch equivalent in total energy deposition to that of $\mathrm{a} \sim 10^{19} \mathrm{eV}$ primary. As the charge traverses the target, an ionization cloud is produced. T-576 sought to measure, for the first time, the radar return from this ionization cloud.

The experimental setup is shown in Figure 1. Two independent data acquisition systems (DAQ) were employed, one was a 4-channel Tektronix $20 \mathrm{GS} / \mathrm{s}$ oscilloscope, and the other was a new, custom 12-channel, 3.2 GS/s DAQ called the SURFv5, based on the LAB4D analog-to-digital converter chip [15]. These DAQ systems will be referred to as "scope" and "SURF", respectively. Three different types of antennas were used in the experiment. First was a pair RFSpace UWB2 Vivaldi-style antenna with a $3 \mathrm{~dB}$ bandwidth from $0.6-6 \mathrm{GHz}$, another was a narrow-band commercial WiFi dish antenna, and last was a set of 13 custom-built printed-circuit-board log-periodic dipole antennas (LPDA) with a $3 \mathrm{~dB}$ bandwidth from 0.95 to $4 \mathrm{GHz}$. These antennas will be referred to as "Vivaldi", "WiFi" and "LPDA", respectively. Twelve LPDAs were used with the SURF, one LPDA was equipped with a $+12 \mathrm{dBi}$ dish and was used with the scope, and the Vivaldi and WiFi antennas were used in various scope channels and also as the transmitting antenna. To produce a measurable reflection such that receiver-side amplification was not needed, a $+50 \mathrm{~dB}$ power amplifier was used on the transmitter. Into this amplifier was an ethernet-controlled signal generator. Both DAQs were also remotely controllable and able to be monitored from the "counting house", which was a small room adjacent to End Station A from which we could monitor the experiment, not being permitted into the experimental hall when the beam was on due to radiation safety concerns. The device in Figure 1 called ICT is the integrating current toroid, and is placed around the end of the beam pipe in order to monitor the beam. As a beam bunch passes through the middle of the ICT, a current is induced in proportion to the amount of charge, giving a very precise measurement of the shot-to-shot beam charge.

The experiment was performed as follows: for one week, we had alternating 12-hour periods of beam and no-beam. During the no-beam periods we made changes to the experimental setup, 
performed quick analysis of data, and planned for beam runs. During beam periods, we took data in a variety of antenna configurations and transmitter frequency and power settings, to best probe the different features of a putative radar signal. Due to the complexities of taking measurements in a radio-noisy, high-background environment, we attempted to maximize our ability to extract signals by probing as many different parameters as possible. Two so-called "smoking guns" turned out to be observable as ways to separate signal from background. These are detailed below.

The analysis presented here was only performed on the scope dataset. Analysis of the SURF dataset, while larger owing to the increased number of channels, will be presented at a later time.

\section{Data analysis}

The analysis for T-576 was challenging due to the high-amplitude backgrounds. These backgrounds, detailed in Ref. [10], are collectively referred to as "beam splash," owing to their overall messy and hard-to-disentangle origin. The beam splash is a combination of sudden-appearance [17], transition radiation [18], and Askaryan radiation, all of which make their way to the receiving antenna at different times and via different direct and reflected paths. Our signal, which was expected at $1 \%$ the amplitude of this beam splash, required a sensitive analysis technique in order to be extracted. Following Ref. [16], we used a matrix decomposition method to extract the signal, and then constructed a set of "null" data, explained below, to verify the analysis method and establish a baseline. We report here on a signal excess consistent with a radar signal.

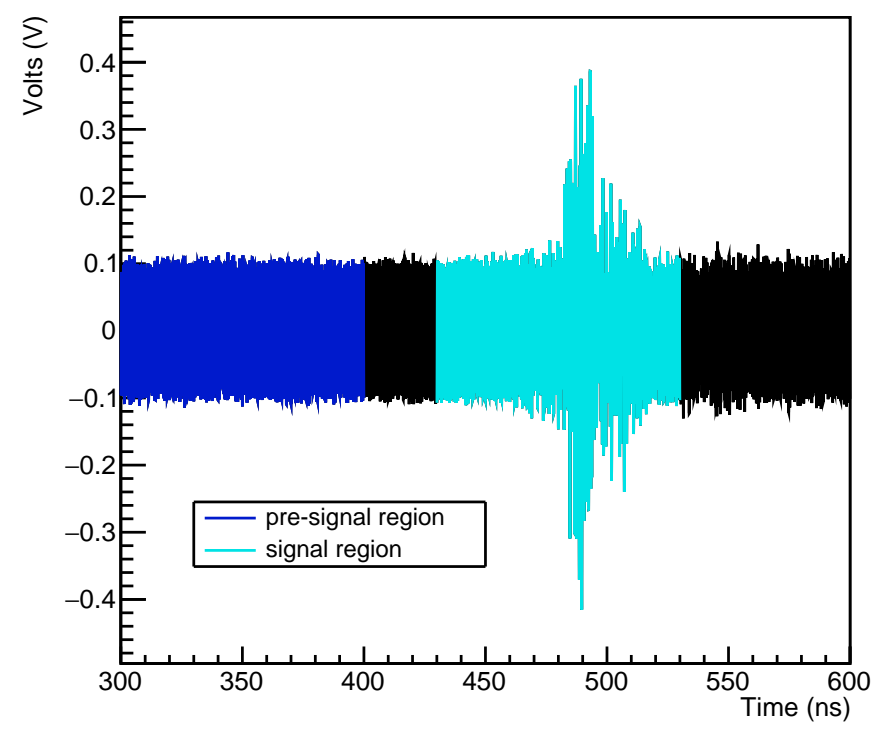

Figure 2: An example full waveform from T-576 with the $\mathrm{CW}$ and beam splash included. Indicated in dark blue (left) is the pre-signal region and the signal region in light blue (right).

\subsection{Null data}

For brevity, we do not include details about exactly how the signal presented here was extracted. Such details can be found in forthcoming (and concurrent) works. We do, however, present 
our results with comparison to a "null" dataset, which is our constructed set of data that ideally contains all of our backgrounds and no signal.

A typical T-576 event is shown in Figure 2. Highlighted in the figure are the beam splash and the pre-signal $\mathrm{CW}$ region. To construct a null waveform, the beam splash/signal region from a transmitter off event is added to the pre-signal CW only region of a different event. The sum of these two is equal to the linear sum of the $\mathrm{CW}$ and beam splash of an actual event in the signal region, but it is guaranteed to be void of a radar signal, because the transmitter was not on at the same time as the shower was produced.

\subsection{Analysis procedure}

For the analysis, we generated a null event for every real event in the experiment, and all analysis was performed simultaneously on both sets. The filtering procedure, using the matrix decomposition routine discussed above, was a blind procedure. A filter was built via matrix decomposition for each event individually, and algorithmically, on the real and null sets, after being tuned on a subset of sub-runs ( $\sim 10 \%$ of the scope dataset).

The radar signal for our setup was expected to be $\mathscr{O}(0.1-1 \mathrm{mV})$ on the background of $\mathscr{O}(100 \mathrm{mV})$, based upon simulations and standard plasma theory. This is at or below the level of noise, and so in may cases, filtration of the data resulted in nothing but noise being evident by eye in the filtered events, even when averaging over hundreds of waveforms. In some configurations however, the resultant signal, when averaged over a sub-run (typically 100-500 events), is large enough to be observed above noise in a spectrogram. We present evidence of scaling of this signal with the output power of the transmitting antenna, one of our "smoking guns". In one particularly favorable configuration with the highest gain antenna and horizontal polarization, such averaging even allows for the extraction of a time-domain waveform above the level of noise, which has good agreement with simulation.

\subsection{Results}

Figure 3 shows a time and frequency representation of the extracted putative signal for a run in which a time-domain waveform was able to be extracted, compared to the signal from a finitedifference time-domain simulation [20], with the exact parameters of T-576, including the HDPE target and transmitting Vivaldi antenna. For this run, the antennas were horizontally polarized, and the transmitter frequency was $2.1 \mathrm{GHz}$. Two procedures were used to generate a time-domain average, and both are shown in Figure 3. The CW transmitter was not phase-locked to the beam, and so the phase of the putative reflection varies from event to event. The absolute timing of the signal remains fixed, however, and so too will the relative phase of a putative reflection to the initial $\mathrm{CW}$ phase. The left panel shows the average of all of the events after phase aligning to the prefiltered, pre-signal CW. The right panel shows the result of averaging when selecting for the highest SNR events. These events had a clear enough signal-region excess that they could be aligned via cross-correlation within the signal region and averaged. In both cases, the alignment is restricted to a half-period $( \pm .24 \mathrm{~ns})$ to disallow artificial elongation of the average. The simulated signal, shown in red, has been scaled up by $2 \mathrm{~dB}$ to account for differences in antenna gains between data and simulation and has been time-aligned to the data. Shown also for reference is the average of the null data for the same run, processed in the same way as the real, which shows no excess. 

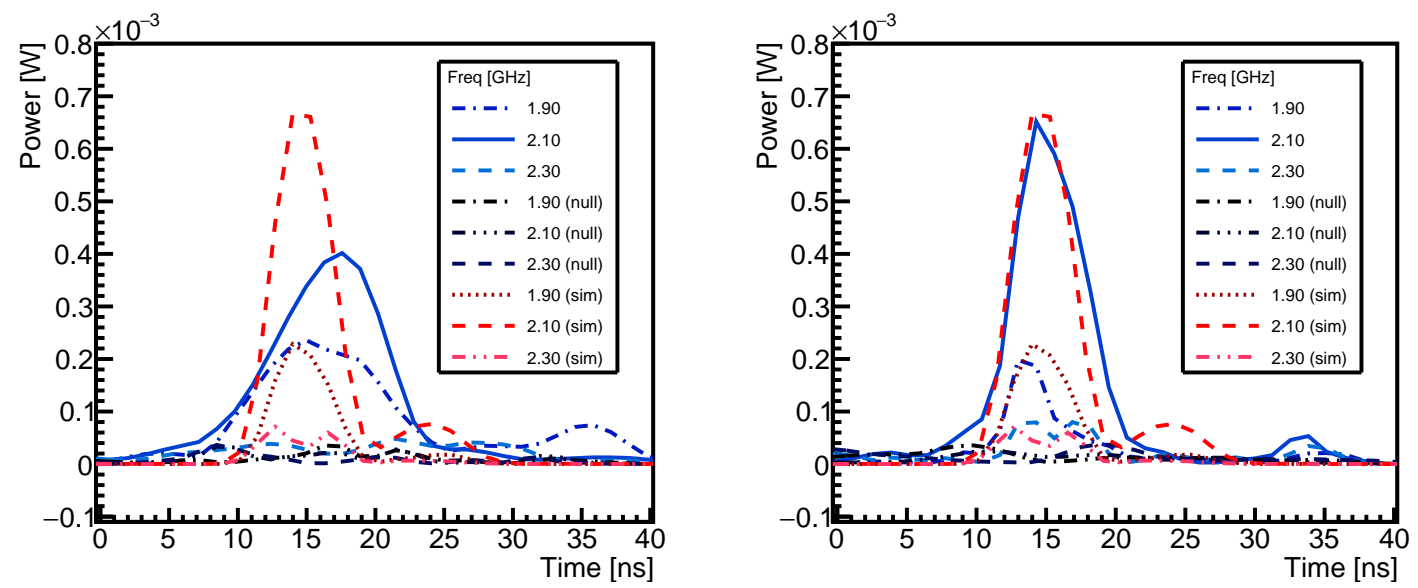

Figure 3: A time and frequency representation of the extracted signal. The left panel shows the power versus time of the extracted signal in T-576 data for different frequency bands, with central frequencies indicated in the legend, with a comparison to the null data (dark blue), and simulation (red) at the same frequencies. This signal is an average of all of the filtered waveforms in this sub-run. The right panel shows the average extracted signal after selecting for high SNR events, as described in the text, with the same comparisons to null and simulation.

Prior to establishing confirmation of the observed excess as a radar signal, further checks must be performed, including a detailed comparison to simulation, where we will explore the signal timing, duration, and spectral content, and an investigation into the averaging procedure discussed above. These will be presented in a forthcoming article. An exhaustive list of the checks performed so far is beyond the scope of this proceeding.

Figure 4 shows simple linear scaling of the power in this extracted signal with the transmitter output power, demonstrating that the extracted signal scales with the transmitter power. In the figure, the $\mathrm{x}$-axis values come from pre-signal, unfiltered $\mathrm{CW}$ measurements, and the $\mathrm{y}$-axis values have been scaled by a constant in order to compare with a $y=x$ trend line.

\section{Conclusion and discussion}

We report on the observation of a signal detected during experiment T-576 at SLAC. We have compared this signal to simulation and found good agreement. Future and concurrent work will further analyze this extracted signal and explicate the checks that have been performed to ensure that the extracted signal is not an analysis artifact or other non-radar feature. Additionally, an analysis of the SURF data, as well as further validation against simulations will be performed.

\section{Acknowledgments}

We thank the personnel at SLAC for providing us with excellent stable beam and a safe, productive work environment. This work was performed in part under SLAC DOE Contract DEAC02-76SF00515, and supported by a US Department of Energy Office of Science Graduate Student Research (SCGSR) award, administered by the Oak Ridge Institute for Science and Education 


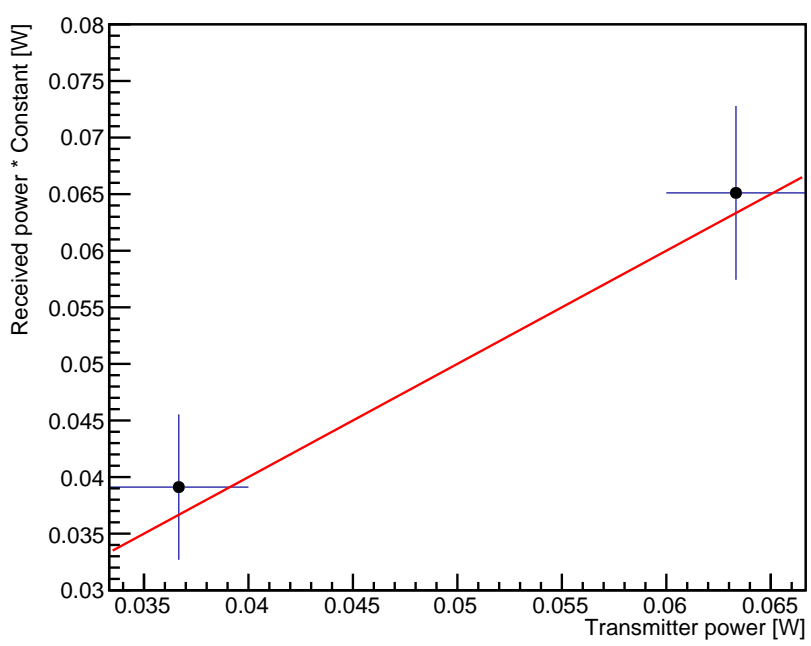

Figure 4: Scaling of the extracted signal with the transmitter output power. The $\mathrm{x}$ axis is measured using CW only, pre-signal, unfiltered data. The y axis has been scaled by a constant to overlay with a $y=x$ trend. Error bars are the standard error on the spread in y-axis values.

for the DOE under contract number DE-SC0014664, as well as by the Flemish Foundation for Scientific Research FWO-12L3715N, the European Research Council under the EU-ropean Unions Horizon 2020 research and innovation programme (grant agreement No 805486), and CCAPP at The Ohio State University.

\section{References}

[1] M. G. Aartsen et al. [IceCube Collaboration], Science 342, 1242856 (2013) doi:10.1126/science.1242856 [arXiv:1311.5238 [astro-ph.HE]].

[2] I. Kravchenko et al. [RICE Collaboration], Astropart. Phys. 19, 15 (2003) doi:10.1016/S0927-6505(02)00194-9 [astro-ph/0112372].

[3] S. W. Barwick, J. Phys. Conf. Ser. 60, 276 (2007) doi:10.1088/1742-6596/60/1/060 [astro-ph/0610631].

[4] P. W. Gorham et al. [ANITA Collaboration], Astropart. Phys. 32, 10 (2009) doi:10.1016/j.astropartphys.2009.05.003 [arXiv:0812.1920 [astro-ph]].

[5] P. Allison et al., Astropart. Phys. 35, 457 (2012) doi:10.1016/j.astropartphys.2011.11.010 [arXiv:1105.2854 [astro-ph.IM]].

[6] G. A. Askar'yan, Sov. Phys. JETP 14, no. 2, 441 (1962) [Zh. Eksp. Teor. Fiz. 41, 616 (1961)].

[7] K. D. de Vries, K. Hanson and T. Meures, Astropart. Phys. 60, 25 (2015) doi:10.1016/j.astropartphys.2014.05.009 [arXiv:1312.4331 [astro-ph.HE]].

[8] S. Prohira and D. Besson, Nucl. Instrum. Meth. A 922, 161 (2019) doi:10.1016/j.nima.2018.12.027 [arXiv:1710.02883 [physics.ins-det]].

[9] K. D. de Vries, P. Coppin, A. O'Murchadha, O. Scholten, S. Toscano and N. van Eijndhoven, arXiv:1802.05543 [astro-ph.HE]. 
[10] S. Prohira et al., arXiv:1810.09914 [hep-ex].

[11] E. D. Bloom et al., Phys. Rev. Lett. 23, 930 (1969). doi:10.1103/PhysRevLett.23.930

[12] D. Saltzberg et al., Phys. Rev. Lett. 86 (2001) 2802 doi:10.1103/PhysRevLett.86.2802 [hep-ex/0011001].

[13] P. W. Gorham et al. [ANITA Collaboration], Phys. Rev. Lett. 99 (2007) 171101 doi:10.1103/PhysRevLett.99.171101 [hep-ex/0611008].

[14] K. Belov et al. [T-510 Collaboration], Phys. Rev. Lett. 116, no. 14, 141103 (2016) doi:10.1103/PhysRevLett.116.141103 [arXiv:1507.07296 [astro-ph.IM]].

[15] J. M. Roberts, E. Oberla, P. Allison, G. S. Varner, S. Spack, B. Fox and B. Rotter, Nucl. Instrum. Meth. A 925, 92 (2019) doi:10.1016/j.nima.2019.01.091 [arXiv:1803.04600 [physics.ins-det]].

[16] A. Bean, J. P. Ralston and J. Snow, Nucl. Instrum. Meth. A 596, 172 (2008) doi:10.1016/j.nima.2008.07.150 [arXiv:1008.0029 [physics.ins-det]].

[17] K. D. de Vries et al., Phys. Rev. D 98, no. 12, 123020 (2018) doi:10.1103/PhysRevD.98.123020 [arXiv:1902.02737 [astro-ph.HE]].

[18] V. L. Ginzburg and I. M. Frank, J. Phys. (USSR) 9, 353 (1945) [Zh. Eksp. Teor. Fiz. 16, 15 (1946)].

[19] K. D. de Vries, S. Buitink, N. van Eijndhoven, T. Meures, A. ÃŞ Murchadha and O. Scholten, Astropart. Phys. 74, 96 (2016) doi:10.1016/j.astropartphys.2015.10.003 [arXiv:1503.02808 [astro-ph.HE]].

[20] Remcom Incorporated, XFdtd, https://www.remcom.com/xfdtd-3d-em-simulation-software 